\title{
Targeted exome sequencing identified a novel USH2A mutation in a Chinese usher syndrome family: a case report
}

\author{
Dongjun Xing ${ }^{\dagger}$, Huaiyu Zhou ${ }^{\dagger}$, Rongguo Yu, Linni Wang, Liying Hu, Zhiqing Li ${ }^{*}$ and Xiaorong Li ${ }^{*}$ (D)
}

\begin{abstract}
Background: Usher syndrome is a disease with a heterogeneous phenotype and genotype. Our purpose was to identify the gene mutation in a Chinese family with Usher syndrome type 2 and describe the clinical features.

Case presentation: A 23-year-old man complained of a 10-year duration of nyctalopia and a 3-year decline in visual acuity of both eyes accompanied by congenital dysaudia. To clarify the diagnosis, the clinical symptoms were observed and analysed in combination with comprehensive ophthalmologic examinations as well as genetic analysis (targeted exome sequencing, TES). A typical clinical presentation of Usher syndrome of the fundus was found, including a waxy yellow-like disc, bone-spicule formations and retinal vessel stenosis. Optical coherence tomography (OCT) and optical coherence tomography angiography (OCTA) showed loss of the ellipsoid zone and a reduction in paracaval vessel density in both eyes. Genetic analysis identified a novel homozygous c.8483_8486del (p.Ser2828*) mutation in USH2A. The mutation resulted in premature termination of translation and caused the deletion of 19 fibronectin type 3 domains (FN3), transmembrane (TM) region and PDZ-binding motif domain, which play an important role in protein binding. After combining the clinical manifestations and genetic results, the patient was diagnosed with Usher syndrome type 2.

Conclusion: We found a novel c.8483_8486del mutation in the USH2A gene through TES techniques. The results broaden the spectrum of mutations in Usher syndrome type 2 and suggest that a combination of clinical information and molecular diagnosis via TES could help Usher syndrome patients obtain a better diagnosis.
\end{abstract}

Keywords: Usher syndrome, Targeted exome sequencing, Mutation, Case report

\section{Background}

Usher syndrome (USH) is an autosomal recessive disease that is characterized by retinitis pigmentosa (RP), sensorineural hearing impairment and vestibule dysfunction. The prevalence of USH is approximately 3.2 to 6.2 per 100,000 individuals [1-4], and it is clinically and genetically heterogeneous. To date, 18 genes and loci

\footnotetext{
*Correspondence: Izhqyk@163.com; xiaorli@163.com

${ }^{+}$Dongjun Xing and Huaiyu Zhou contributed equally to this work. Tianjin Key Laboratory of Retinal Functions and Diseases, Tianjin International Joint Research and Development Centre of Ophthalmology and Vision Science, Eye Institute and School of Optometry, Tianjin Medical University Eye Hospital, 251 Fukang Road, Tianjin 300384, China
}

have been associated with USH (RetNet [https://sph.uth. edu/retnet]; August 2020). Among them, 15 genes were identified as causative genes. USH can be divided into three types according to the age of onset, the severity of visual and hearing impairment and vestibule dysfunction. However, because the genetic manifestations are currently not well understood, the rate of missed diagnosis (4\%) is high in Asia, especially in China [5].

Deafness occurs early in patients with USH1, and their abnormal visual function is easily ignored. In patients with USH2 and USH3, visual function and hearing abnormalities are gradually progressive. Accurate clinical and molecular diagnoses are the basis of prognosis

C The Author(s). 2020 Open Access This article is licensed under a Creative Commons Attribution 4.0 International License, which permits use, sharing, adaptation, distribution and reproduction in any medium or format, as long as you give appropriate credit to the original author(s) and the source, provide a link to the Creative Commons licence, and indicate if changes were made. The images or other third party material in this article are included in the article's Creative Commons licence, unless indicated otherwise in a credit line to the material. If material is not included in the article's Creative Commons licence and your intended use is not permitted by statutory regulation or exceeds the permitted use, you will need to obtain permission directly from the copyright holder. To view a copy of this licence, visit http://creativecommons.org/licenses/by/4.0/ The Creative Commons Public Domain Dedication waiver (http://creativecommons.org/publicdomain/zero/1.0/) applies to the data made available in this article, unless otherwise stated in a credit line to the data. 
prediction, treatment selection and genetic counselling. Targeted exome sequencing (TES) provides a new opportunity to reveal the genetic defects in USH patients [6]. Here, we screened 381 inherited retinal disease (IRD)-related genes in an USH2 family and identified a novel c.8483_8486del (p.Ser2828*) mutation in the USH $2 A$ gene.

\section{Case presentation}

A 23-year-old man visited our clinic and had suffered from deafness from childhood with occasional dizziness, nyctalopia for 10 years and visual acuity decline of both eyes for nearly 3 years. Previously, he was diagnosed with "sensorineural deafness" by an otorhinolaryngologist. The patient and his family members gave informed consent for the study, which was approved by the Ethics Committee of Tianjin Medical University Eye Hospital (Tianjin, China). Then, peripheral venous blood samples were collected for TES and Sanger sequencing. For the clinical diagnosis, we performed a comprehensive ocular examination that included determination of bestcorrected visual acuity (BCVA), slit-lamp examination, visual field tests, optical coherence tomography (OCT), optical coherence tomography angiography (OCTA), ultra-wide field fundus photography, and fundus autofluorescence (FAF).

\section{Methods}

\section{DNA library preparation}

Genomic DNA was extracted from peripheral blood leukocytes of the patient and his family members using a DNA Extraction Kit (TIANGEN, Beijing, China) following the manufacturer's instructions. The DNA was quantified with a Nanodrop 2000 (Thermal Fisher Scientific, DE). A minimum of $3 \mu \mathrm{g}$ of DNA was used for the indexed Illumina libraries according to the manufacturer's protocol (My Genostics, Inc., Beijing, China). DNA fragments with sizes ranging from $350 \mathrm{bp}$ to 450 bp and those including the adaptor sequences were selected for the DNA libraries.

\section{Targeted gene capture and sequencing}

Next, 381 known genes associated with IRDs, including USH (Additional file 2: Table S2), were selected by a gene capture strategy using the GenCapCustom Enrichment Kit (My Genostics Inc., Beijing, China) following the manufacturer's protocol. The biotinylated capture probes were designed to tile all of the exons with nonrepeated regions. Sequencing was performed on an Illumina HiSeq 2000 sequencer (Illumina, San Diego, CA, USA) for paired-end reads of $150 \mathrm{bp}$.

\section{Bioinformatics analysis}

Following sequencing, raw image files were processed using Bcl2Fastq software (Bcl2Fastq, Illumina, Inc.) for base calling and raw data generation. Low-quality variations (score $\geq 20$ ) were filtered out. The clean reads were then aligned to the reference human genome using Short Oligonucleotide Analysis Package (SOAP) aligner software (SOAP2.21; soap.genomics.org.cn/soapsnp.html) (hg19). After removing polymerase chain reaction (PCR) duplicates using the Picard program, single nucleotide polymorphisms (SNPs) were determined using the SOAP SNP program, and the deletions and insertions (InDels) were detected using Genome Analysis Toolkit software 3.7. Subsequently, we annotated the identified SNPs and InDels with the Exome-assistant program (http://122.22 8.158.106/exomeassistant) and viewed the short read alignment using MagicViewer to confirm the candidate SNPs and InDels. Non-synonymous variants were evaluated for pathogenicity using the Sorting Intolerant From Tolerant [SIFT; (http://sift.jcvi.org/)] and PolyPhen (http://genetics.bwh.harvard.edu/pph2/) tools as well as Protein Analysis Through Evolutionary Relationships (PANTHER; www.pantherdb.org) and Pathogenic Mutation Prediction (Pmut; http://mmb.pcb.ub.es/PMut/).

\section{Expanded validation and protein function prediction}

Genomic DNA of the proband was subjected to TES. Filtered candidate variants identified by an Illumina HiSeq 2000 sequencer were confirmed by Sanger sequencing. The coding exons containing the detected mutations were amplified using Ex Tag DNA polymerase (Takara, Dalian). The purified PCR samples were sequenced using an ABI PRISM 3730 genetic analyser (Applied Biosystems; Thermo Fisher Scientific, Inc.), and then sequence traces were analysed with Mutation Surveyor (Softgenetics, PA). The mutation was confirmed in the family members by the same procedure. Multiple sequence alignments were performed using ClustalW2 with the default setting (http://www.ebi.ac.uk/Tools/clustalw2/). Protein structures were determined by SMART (http://smart.emblheidelberg.de). The variation in the 3D structure of the protein caused by gene mutation was analysed using Protein Data Bank (PDB) and the homology modelling software Swiss-Model. All genomic DNA samples were collected after obtaining informed consent.

\section{Results}

\section{Clinical findings}

A 23-year-old man presented a 10-year history of deafness and poor night vision. His BCVA was 0.6/1.0 (R/L). His parents had a consanguineous marriage, and his grandparents were deceased, but they were healthy according to their past medical history and eye conditions. In addition, his parents and his sister were unaffected 


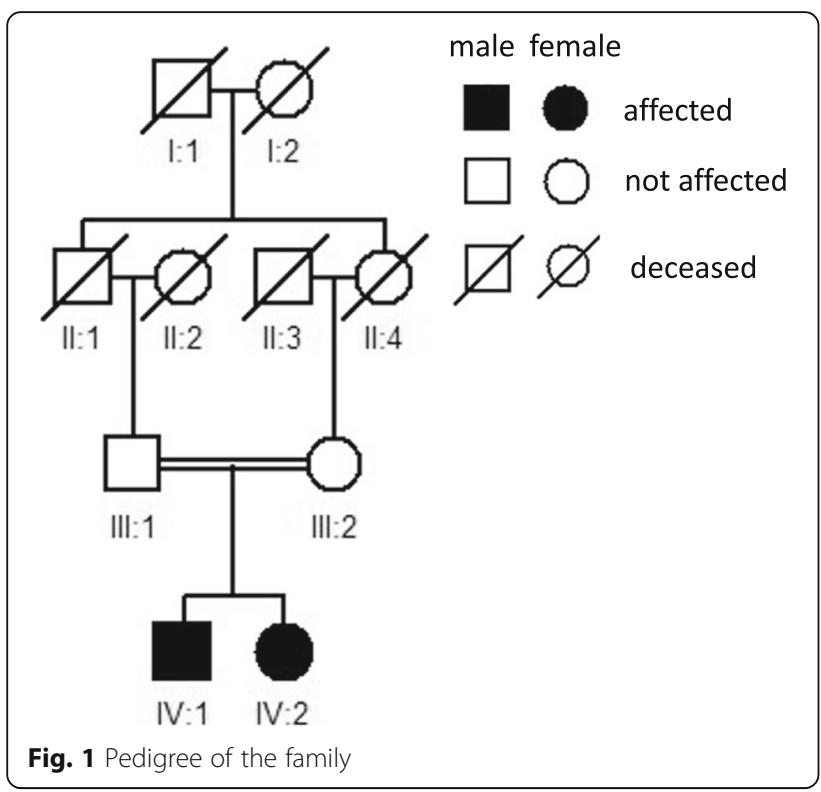

(Fig. 1). Ophthalmologic investigations were performed to better clarify the proband's condition. Slit-lamp examination showed that the anterior segment of the eyes was normal. The fundus had typical RP characteristics, including the appearance of a waxy yellow-like disc, a large amount of osteoblast-like pigmentation, and tapering of the retinal vessels, which was obvious in the arteries (Fig. 2a). An abnormal parafoveal ring of increased autofluorescence was observed in ultra-wide-angle images, and a ring-like hypoautofluorescence region was observed around the macula and optic disc on FAF imaging (Fig. 2b). Examination with an Octopus perimeter device showed a tubular visual field in both eyes (Fig. 2c). We also found decreased retinal thickness and absence of the ellipsoid zone in the macula (Fig. 2d). Macular OCTA revealed an enlarging foveal avascular zone (FAZ) in the superficial capillary plexus and deep capillary plexus, while macular vascular flow density was also decreased (Fig. 2e).

\section{Genetic and molecular analysis}

DNA extracted from the peripheral blood was subjected to TES (Additional file 1: Table S1). Genetic

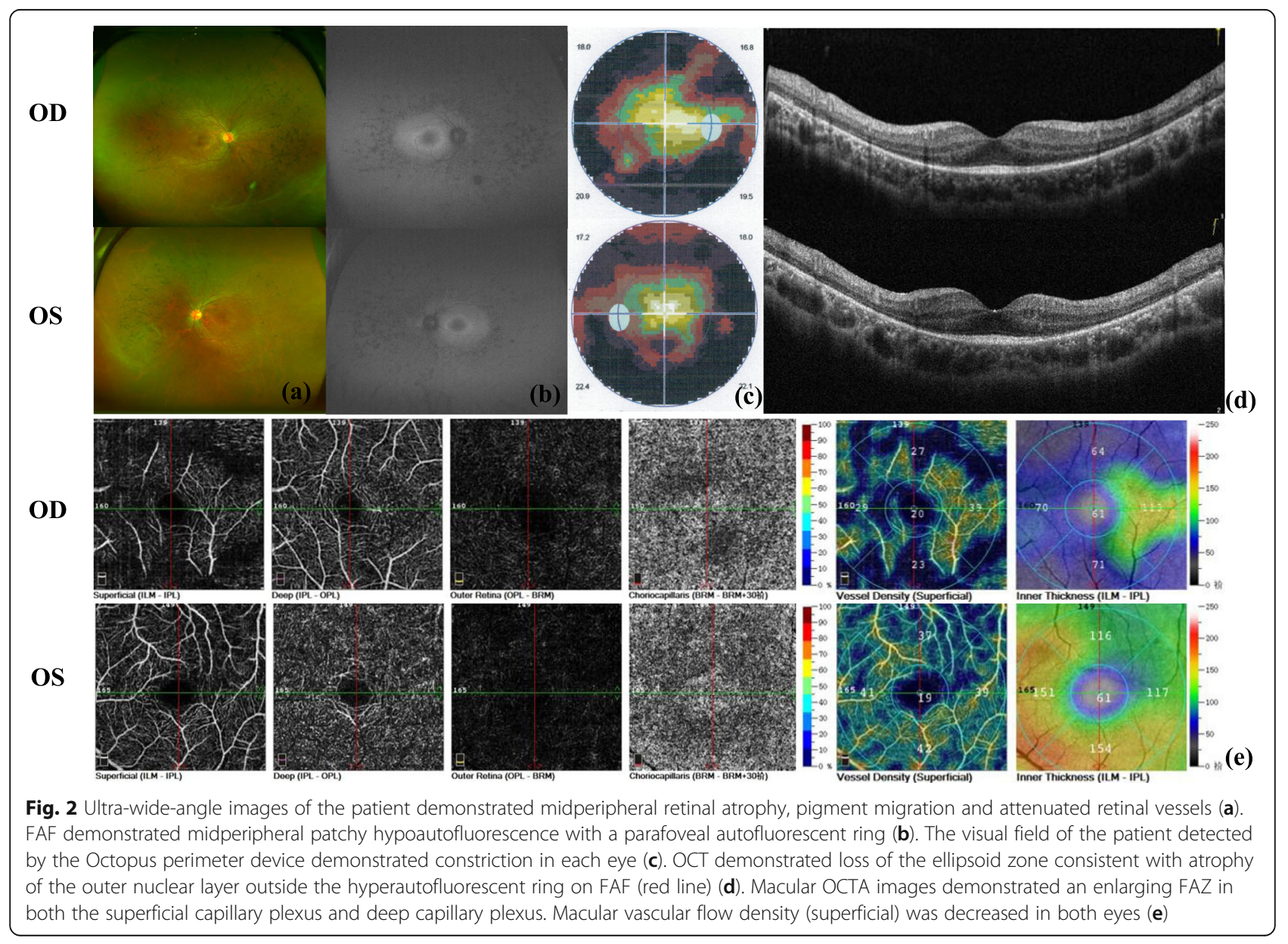


tests showed that the patient had a novel mutation (c.8483_8486del) in the USH2A gene. Moreover, DNA samples extracted from the proband's sister and parents were used for Sanger sequencing. Genetic cosegregation analysis was confirmed in this family (Fig. 3a). A model structure for USH2A was generated via homology modelling (Fig. 3b). The mutation resulted in premature termination of translation, and the stop-gain variant was predicted to remove 2375 amino acids from the encoded protein, which would result in truncation of the $\alpha / \beta$-hydrolase domain (Fig. 3c) and may change the overall function of the folded state of the protein (Fig. 3d).

\section{Discussion and conclusions}

We reported the case of a 23-year-old patient who presented a series of typical clinical features with a novel homozygous mutation, p.Ser2828* (rs1199684717), in USH2A, a gene responsible for USH2 (OMIM:276901). The frequency of the mutation is 0.000004 in the

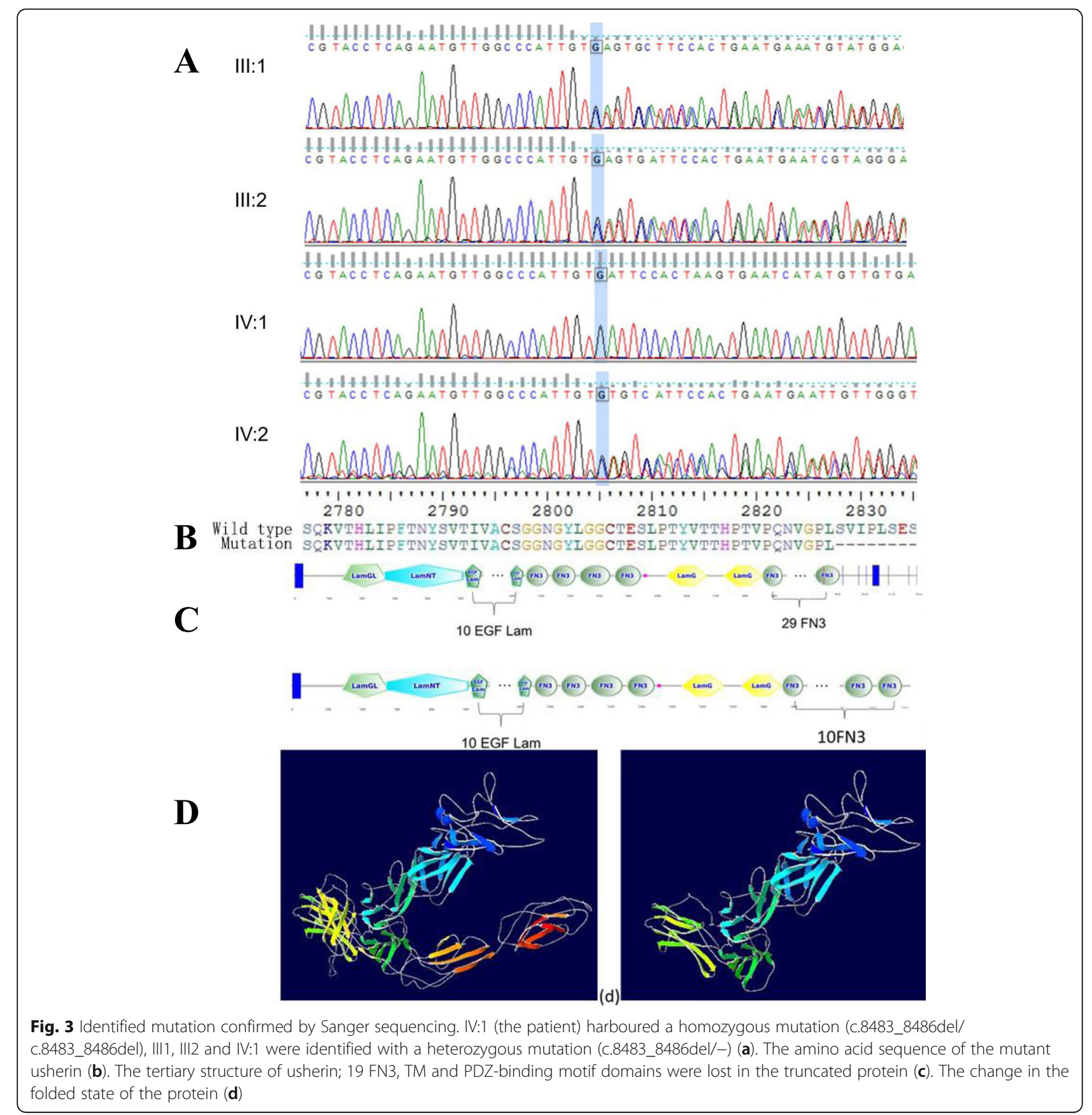


Genome Aggregation Database, and it was found in a heterozygous state in one European non-Finnish individual. Mutations in $U S H 2 A$ are associated with USH2, which is responsible for almost $50 \%$ of USH cases [7].

$\mathrm{USH} 2 \mathrm{~A}$ codes two alternatively spliced isoforms of usherin. The short $\sim 170 \mathrm{kDa}$ isoform a, consisting of 21 exons, is regarded as an extracellular protein. The fulllength $\sim 580 \mathrm{kDa}$ isoform $\mathrm{b}$ is a complex transmembrane protein composed of three regions: a large extracellular region consisting of an $\mathrm{N}$-terminal signal peptide, laminin G-like domain (LamGL), laminin domain $\mathrm{N}$-terminal (LamNT), laminin-type EGF-like modules (EGF-Lam), fibronectin type III (FN3) repeats, laminin G domains (LamG); a transmembrane region (TM); and a cytoplasmic C-terminal domain containing a PDZ-binding motif $[8,9]$. Usherin is distributed in the periciliary membrane complex and synapse in photoreceptors. All USH1 and USH2 proteins are organized as protein networks by the scaffold proteins harmonin (USH1C), whirlin (USH2D) and SANS (USH1G). Usherin (USH2A) and VLGR1b (USH2C) are part of the links that are intracellularly attached to the scaffold proteins. However, during the differentiation of the hair bundle, both USH1 and USH2 proteins contribute to the formation of side links located at the tip and the base of the stereocilia, respectively. They exist in multiprotein complexes that work together as molecular networks to anchor them to the stereocilia actin filaments [10-14].

The homozygous mutation (p.Ser2828*) in USH2A caused premature termination of translation, and as a result, 19 FN3, TM and PDZ-binding motif domains were deleted. FN3 plays a key role in cell adhesion, cell morphology, thrombosis, cell migration, and embryonic differentiation as well as pathophysiologic processes such as angiogenesis and vascular remodelling [15]. A TM domain is present at the base of differentiating stereocilia and causes the mechanosensitive hair bundles to be receptive to sound. PDZ-binding motif domains provide the anchoring of interstereocilia lateral links to the F-actin core of stereocilia [16]. In this regard, we suppose that the absence of these domains corresponding to the incompleteness of usherin might have affected the process of differentiation and maturation of the stereocilia, resulting in a milder dysmorphic phenotype of the stereocilia. Several missense hotspots have been associated with the pathogenesis of FN3 in usherin [17], which supports our hypothesis. However, this pathway needs to be confirmed by molecular experiments in the future.

Whole-genome sequencing (WGS), whole-exome sequencing (WES) and TES are three major methodologies for molecular diagnosis of IRDs. WGS is useful for detecting copy number and structural variations [18]. WES is especially useful for identifying novel IRD-related genes. TES is an accurate, rapid and cost- effective approach for screening of multiple genes [19], but it still has some major limitations, such as detecting variants in low-depth regions and copy number variations $[18,20]$. Because of their high cost, both of WGS and WES are less widely used than TES. TES is suitable for molecular diagnosis of USH. Because of the great diversity of various types of pathogenic genes and the frequent occurrence of new mutations, array-based diagnosis often can not accurately reflect pathogenicity. Pathogenic USH genes have many subtypes and numerous exons. At present, more than 400 coding exons have been identified [21]. Therefore, a higher diagnosis rate can be obtained using a sequence-based diagnosis method.

Here, we report a novel homozygous mutation, c.8483_8486del, in the USH2A gene identified through TES techniques. The mutation truncated $U S H 2 A$ gene translation, and 19 FN3, TM and PDZ-binding motif domains were lost, which influenced the function of stereocilia. We broadened the spectrum of mutations of this disease and provided a new locus for gene therapy of USH. The combination of molecular diagnosis by TES and clinical information can help USH patients obtain more accurate diagnoses.

\section{Supplementary Information}

The online version contains supplementary material available at https://doi. org/10.1186/s12886-020-01711-7.

Additional file 1: Table S1. 381 known genes associated with IRD.

Additional file 2: Table S2. The TES data of the proband.

Additional file 3.

Additional file 4.

Additional file 5 .

Additional file 6 .

\section{Abbreviations}

USH: Usher syndrome; OCT: Optical coherence tomography; OCTA: Optical coherence tomography angiography; IRD: Inherited retinal disease; FN3: Fibronectin type 3; TES: Targeted exome sequencing; RP: Retinitis pigmentosa; BCVA: Best-corrected visual acuity; FAF: Fundus

autofluorescence; SOAP: Short Oligonucleotide Analysis Package; SNPs: Single nucleotide polymorphisms; PCR: Polymerase chain reaction; PDB: Protein Data Bank; FAZ: Foveal avascular zone; LamGL: Laminin G-like domain; LamNT: Laminin domain N-terminal; EGF-Lam: Laminin-type EGF-like modules; LamG: Laminin G domains

\section{Acknowledgements}

We thank all of the family members for their participation in this study.

Authors' contributions

$X \mathrm{~L}$ and $\mathrm{ZL}$ conceived the idea and take responsibility for the integrity of the data. DX and $\mathrm{HZ}$ collected the samples, performed data analyses and wrote the manuscript. Both authors also contributed equally to this work. RY, LW and $\mathrm{LH}$ performed the experiments. All authors have read and approved the final manuscript.

\section{Funding}

This study was supported by the Tianjin Clinical Key Subject Construction Project (Award number: TJLCZDXKQ022; Recipient: Dongjun Xing) in China 
for design of the study and the Natural Science Foundation of Tianjin City (Award number: 17JCYBJC27200; Recipient: Zhiqing Li) for analysis of data.

\section{Availability of data and materials}

All data are fully available without restriction. The TES data of the proband in Additional file 1: Table S1; The data of Sanger sequencing: III, III2, IV1, IV2.

\section{Ethics approval and consent to participate}

This study adhered to the tenets of the Declaration of Helsinki and was approved by the Institutional Review Board of Tianjin Medical University Eye Hospital (Tianjin, China), Number: 2019KY-02. Written informed consent for participation was obtained from the patients.

\section{Consent for publication}

Written informed consent was obtained from the patient and all family relatives genetically tested for participation and the publication according to Ethics Committee Regulations.

\section{Competing interests}

The authors declare that they have no competing interests.

Received: 23 December 2019 Accepted: 29 October 2020

Published online: 10 December 2020

\section{References}

1. Blanco-Kelly F, Jaijo T, Aller $E$, et al. Clinical aspects of usher syndrome and the USH2A gene in a cohort of 433 patients. JAMA Ophthalmol. 2015;133(2): 157-64.

2. Cohen M, Bitner-Glindzicz M, Luxon L. The changing face of usher syndrome: clinical implications. Int J Audiol. 2007;46(2):82-93.

3. Hartel $B P$, Löfgren $M$, Huygen $P L$, et al. A combination of two truncating mutations in USH2A causes more severe and progressive hearing impairment in usher syndrome type lla. Hear Res. 2016;339:60-8.

4. Hope $\mathrm{Cl}$, Bundey S, Proops D, Fielder AR. Usher syndrome in the city of Birmingham--prevalence and clinical classification. Br J Ophthalmol. 1997; 81(1):46-53.

5. Pehere NK, Khanna RC, Marlapati R, Sannapaneni K. Prevalence of ophthalmic disorders among hearing-impaired school children in Guntur district of Andhra Pradesh. Indian J Ophthalmol. 2019;67(4):530-5.

6. Huang XF, et al. Targeted exome sequencing identified novel USH2A mutations in usher syndrome families. PLoS One. 2013;8(5):e63832.

7. Jouret G, Poirsier C, Spodenkiewicz M, et al. Genetics of usher syndrome: new insights from a meta-analysis. Otol Neurotol. 2019:40(1):121-9.

8. Méndez-Vidal C, González-Del Pozo M, Vela-Boza A, et al. Whole-exome sequencing identifies novel compound heterozygous mutations in USH2A in Spanish patients with autosomal recessive retinitis pigmentosa. Mol Vis. 2013:19(1):2187-95.

9. Pierrache $\mathrm{LH}$, Hartel BP, van Wijk E, et al. Visual prognosis in USH2Aassociated retinitis Pigmentosa is worse for patients with usher syndrome type lla than for those with Nonsyndromic retinitis Pigmentosa. Ophthalmology. 2016:123(5):1151-60.

10. Bonnet C, El-Amraoui A. Usher syndrome (sensorineural deafness and retinitis pigmentosa): pathogenesis, molecular diagnosis and therapeutic approaches. Curr Opin Neurol. 2012;25(1):42-9.

11. Cosgrove D, Zallocchi M. Usher protein functions in hair cells and photoreceptors. Int J Biochem Cell Biol. 2014;46:80-9.

12. Sorusch $\mathrm{N}$, et al. Usher syndrome protein network functions in the retina and their relation to other retinal ciliopathies. Adv Exp Med Biol. 2014;801: 527-33.

13. Mathur $P$, Yang J. Usher syndrome: hearing loss, retinal degeneration and associated abnormalities. Biochim Biophys Acta. 2015;1852(3):406-20.

14. Kremer H, van Wijk E, Marker T, Wolfrum U, Roepman R. Usher syndrome: molecular links of pathogenesis, proteins and pathways. Hum Mol Genet 2006;15 Spec No 2:R262-70.

15. Maurer LM, Ma W, Mosher DF. Dynamic structure of plasma fibronectin. Crit Rev Biochem Mol Biol. 2015;51(4):213-27.

16. Adato A, Lefèvre G, Delprat B, Michel V, Michalski N, Chardenoux S, et al. Usherin, the defective protein in usher syndrome type IIA, is likely to be a component of interstereocilia ankle links in the inner ear sensory cells. Hum Mol Genet. 2005;14(24):3921-32. https://doi.org/10.1093/hmg/ddi416.
17. Baux D, Blanchet C, Hamel C, et al. Enrichment of LOVD-USHbases with 152 USH2A genotypes defines an extensive mutational spectrum and highlights missense hotspots. Hum Mutat. 2014;35(10):1179-86.

18. Huang XF, Mao JY, Huang ZQ, et al. Genome-wide detection of copy number variations in unsolved inherited retinal disease. Invest Ophthalmol Vis Sci. 2017:58(1):424-9.

19. Huang XF, Wu J, Lv JN, Zhang X, Jin ZB. Identification of false-negative mutations missed by next-generation sequencing in retinitis pigmentosa patients: a complementary approach to clinical genetic diagnostic testing. Genet Med. 2015;17(4):307-11.

20. Huang XF, Huang F, Wu KC, et al. Genotype-phenotype correlation and mutation spectrum in a large cohort of patients with inherited retinal dystrophy revealed by next-generation sequencing. Genet Med. 2015;17(4): 271-8.

21. Jiang $L$, Liang $X, L i Y$, et al. Comprehensive molecular diagnosis of 67 Chinese usher syndrome probands: high rate of ethnicity specific mutations in Chinese USH patients. Orphanet J Rare Dis. 2015;10:110.

\section{Publisher's Note}

Springer Nature remains neutral with regard to jurisdictional claims in published maps and institutional affiliations.
Ready to submit your research? Choose BMC and benefit from:

- fast, convenient online submission

- thorough peer review by experienced researchers in your field

- rapid publication on acceptance

- support for research data, including large and complex data types

- gold Open Access which fosters wider collaboration and increased citations

- maximum visibility for your research: over $100 \mathrm{M}$ website views per year

At $\mathrm{BMC}$, research is always in progress.

Learn more biomedcentral.com/submissions 